Ryszard Biernat

Tarnów

\title{
Spécificité pastorale de la personnalité de Jean-Paul II selon Jean-Marie Lustiger
}

\author{
Specific Pastoral Personality of John Paul II \\ according to Jean-Marie Lustiger
}

\section{Abstract}

Jean-Marie Lustiger admired John Paul II and he was devoted to him. They both had similar origin. The Cardinal of Paris often accompanied the pope in his pastoral visits. He perceived John Paul II as the man of Providence. He was convinced that God had prepared him to this role. Jean-Marie Lustiger emphasized the attitudes of John Paul II that attracted the attention and admiration of observers. For instance: pray, holiness, attentive pastor and good relation with young people. Jean-Marie Lustiger as an attentive observer also focused his attention on the pastoral methods of John Paul II. In his utterances he often perceived the following methods: each person was important, defense of person's dignity, the ability to speak with each man, modern language, importance of the pastoral visits and sui generis spiritual pedagogic. Jean-Marie Lustiger noticed radical change of perspective in preaching and in pastoral action of John Paul II. According to him the pope perceived the challenges of the modern times, evaluated needs of the humanity and gave the appropriate answers. For these he did not choose politics, sociology or even anthropology as a reflection but addressed to the hurt human who was redeem by Jesus Christ.

\section{Keywords}

John Paul II, Jean-Marie Lustiger, pastor, pastoral attitudes, pastoral methods. 


\section{Préface}

La béatification de Jean-Paul II, le $1^{\text {er }}$ mai 2011, était une occasion pour beaucoup de chercheurs de se pencher sur la richesse des pensées et des attitudes du bienheureux. Cet article est un essai d'esquisse de la spécificité pastorale de Jean-Paul II vue par le cardinal Jean-Marie Lustiger. Le cardinal de Paris décédé en 2007, grâce à son parcours et à sa personnalité s'inscrivait dans l'histoire de la vie de Jean-Paul II et il s'appuyait sur son enseignement en essayant de suivre son dynamisme pastoral. Tous les deux étaient proches l'un de l'autre. Ils étaient entre autres liés par leurs racines polonaises, par le phénomène juif, par les épreuves de la Shoa, et surtout par l'amour pour l'homme racheté par Jésus le Christ. L'archevêque de Paris avait pour Jean-Paul II une admiration profonde et un attachement de plus en plus personnel au fil des ans et il est permis d'estimer que lui aussi jouissait de sa part d'une estime remarquable ${ }^{1}$.

Dans cet article seront présentés quatre petits chapîtres dans lesquels nous décrirons la personne de Jean-Paul II, en nous appuant sur les allocutions, les remarques et les articles de Jean-Marie Lustiger à l'occasion des différents événements de la vie de l'Eglise, surtout à l'occasion de JMJ à Paris et des visites pastorales du Saint Père en France. Dans le premier chapître nous allons rappeler l'aspect providentiel de l'élection de Karol Wojtyła au Siège Apostolique. Dans le deuxième chapître nous soulignerons les attitudes humaines et spirituelles mais surtout attirantes du pape. Le troisième chapître contiendra le paysage des différentes formes pastorales utilisées par Jean-Paul II. Dans le quatrième chapître, qui sera en même temps une forme de résumé, nous essaierons de montrer la source d'où jaillissait l'efficacité de son ministère.

\section{Un homme providentiel}

J.-M. Lustiger, en constatant un basculement complet de la civilisation mondiale, estimait l'élection de Karol Wojtyła au Siège de l'Evêque de Rome comme providentielle. Il était convaincu que l'archevêque de Cracovie était un homme préparé par la Providence pour être à la tête de l'Eglise, parce que, grâce à son ministère et à sa participation au Concile Vatican II, l'Eglise a pu le vivre comme une oeuvre de profonde conversion et comme un appel de l'Esprit

${ }^{1}$ Voir: J. Duchesne, Présentation, en: Jean-Marie Lustiger, témoin de Jean-Paul II. Articles, conférences, entretiens, homélies... 1980-2007, Paris 2011, édition Parole et Silence, Communio, p. 9. 
Saint à renouveler la vie de la foi, pour annoncer l'Evangile à ce monde qui propage la civilisation de la mort. Jean-Paul II, grâce à son ministère à la fin du $\mathrm{XX}$-ème siècle perçu par des observateurs comme dur, violent, cruel et soumis à d'horribles déterminismes, a su susciter la liberté de l'Evangile qui a fait basculer des contraintes que les hommes croyaient inamovibles ${ }^{2}$. Aux yeux de Jean-Marie Lustiger Jean-Paul II était un mystique dont la Providence a fait un homme d'action qui comprennait bien les événements dans la continuité de l'histoire du Salut ${ }^{3}$. Le cardinal de Paris savait que l'élection de Karol Wojtyła au Siège Apostolique n'avait pas été, comme le voyait beaucoup de journalistes, un coup de la CIA, mais une manifestation de la puissance de la Sagesse de Dieu qui régit toutes choses avec douceur et force ${ }^{4}$. Cet aspect providentiel a eu ses origines dans l'engagement de Karol Wojtyła dans les travaux du Concil Vatican II. Jean-Marie Lustiger a souligné à plusieurs reprises le rôle important de JeanPaul II dans le déroulement de Vatican II, en le nommant «l'artisant majeur» de ce concile. Dès le début de son pontificat Jean-Paul II a orienté sa pastorale vers la mise en oeuvre de la catholicité de l'Eglise et il puisait toujours dans les élaborations du Vatican II.

\section{Attitudes}

Les différentes attitudes, souvent non-conventionnelles, de Jean-Paul II ont beaucoup impressionné les personnes qui ont eu la grâce d'être avec le Saint Père. La plus importante de ses attitudes était peut-être sa prière. Elle exprimait spirituellement l'acte pastoral. A l'exemple de saint Paul il s'offrait au Père avec tous les dons reçus, en union avec le Christ Unique Pasteur. Jean-Marie Lustiger voyait dans cette prière un résumé d'une attitude que chaque Eglise et chaque chrétien devrait faire sienne ${ }^{5}$. Il a été frappé par la force de l'egagement de JeanPaul II, de son courage et de sa disponibilité. Il ne pensait jamais à lui-même, mais toujours à sa mission, et il était convaincu que cela découlait justement de

${ }^{2}$ Voir: J.-M. Lustiger, 16 octobre: „Je ne sais pourquoi, j'ai éprouvé une immense joie”, en: Jean-Marie Lustiger, témoin de Jean-Paul II. Articles, conférences, entretiens, homélies... 19802007, op. cit., p. 13.

${ }^{3}$ Voir: J.-M. Lustiger, Le „nouvel âge de l'humanité“, en: Jean-Marie Lustiger, témoin de JeanPaul II. Articles, conférences, entretiens, homélies... 1980-2007, op. cit., p. 89-90.

${ }^{4}$ Voir: J.-M. Lustiger, Le pape du concile, en: Jean-Marie Lustiger, témoin de Jean-Paul II. Articles, conférences, entretiens, homélies... 1980-2007, op. cit., p. 109.

${ }^{5}$ Voir: J.-M. Lustiger, Successore di Petro e imitatore di Paulo, en: Giovanni Paulo II pellegrino per il Vangelo, ouvrage collectif, Milan 1988, edizioni Paoline - editrice SAIE, p. 121. 
cette prière ${ }^{6}$. Jean-Paul II était l'apôtre de la prière qui contenait le secret de son action en tant que pasteur présent dans l'histoire humaine et en tant que serviteur de Dieu?

La sainteté de Jean-Paul II était pour Jean-Marie Lustiger évidente. Au cours sa mission de successeur de Pierre il a été physiquement agressé et il était persuadé, que sa guérison était de l'ordre du don de Dieu et il vivait tout cela comme quelque chose de normal dans l'accomplissement de sa mission ${ }^{8}$. JeanPaul II, comme tous les saints, a suivi le même chemin que tous les hommes, mais il marchait plus vite. Son rôle est maintenant de se retourner et de les prendre par la main en les encourageant sur ce chemin'.

Jean-Paul II, selon les dons reçus de la nature et de la grâce, laissait transparaître avec clarté ce qu'est le pasteur. Jean-Marie Lustiger a souligné qu'au moment d'un contact avec le Saint Père qui était toujours très familier, on pouvait s'adresser à lui, non comme à un souverain, mais comme au premier témoin de la foi de l'Eglise et au serviteur de la charité ${ }^{10}$. Il n'exerçait pas l'autorité à la manière d'un administrateur, mais comme un visionnaire audacieux et réaliste ${ }^{11}$. Il n'était pas un séducteur ni une vedette médiatique, mais il savait communiquer, parce que, dans la foi, il s'exprimait toujours comme un frère qui parlait avec son coeur à un frère. Il ne cherchait pas à devenir le maître ni le «gourou» du monde, mais il s'efforçait d'y annoncer la Bonne Nouvelle ${ }^{12}$. Il n'intervenait pas comme négociateur, mais comme annonciateur et il ne proposait pas habilement le minimum, mais prophétiquement le maximum ${ }^{13}$.

${ }^{6}$ Voir: J.-M. Lustiger, Dieu dispose, en: Jean-Marie Lustiger, témoin de Jean-Paul II. Articles, conférences, entretiens, homélies... 1980-2007, op. cit., p. 144.

${ }^{7}$ Voir: J.-M. Lustiger, Les JMJ et le Jubilé de l'an 2000, en: Jean-Marie Lustiger, témoin de Jean-Paul II. Articles, conférences, entretiens, homélies... 1980-2007, op. cit., p. 145.

${ }^{8}$ Voir: J.-M. Lustiger, Ce que nous vivons avec lui, en: Jean-Marie Lustiger, témoin de JeanPaul II. Articles, conférences, entretiens, homélies... 1980-2007, op. cit., p. 61.

${ }^{9}$ Voir: J.-M. Lustiger, Ce que nous vivons avec lui, en: Jean-Marie Lustiger, témoin de JeanPaul II. Articles, conférences, entretiens, homélies... 1980-2007, op. cit., p. 63.

${ }^{10}$ Voir: J.-M. Lustiger, Le successeur de Pierre, porteur du charisme de Paul, en: Le pape en France, Jean-Marie Lustiger, témoin de Jean-Paul II. Articles, conférences, entretiens, homélies... 1980-2007, op. cit., p. 41.

${ }^{11}$ Voir: J.-M. Lustiger, Le „nouvel âge de l'humanité“, en: Jean-Marie Lustiger, témoin de Jean-Paul II. Articles, conférences, entretiens, homélies... 1980-2007, op. cit., p. 90.

${ }^{12}$ Voir: J.-M. Lustiger, La puissance visionnaire du serviteur de la Vérité, „Géopolitique“ (1997) $n^{\circ} 58$, p. 6-7.

${ }^{13}$ Voir: J.-M. Lustiger, Le „nouvel âge de l'humanité“, en: Jean-Marie Lustiger, témoin de Jean-Paul II. Articles, conférences, entretiens, homélies... 1980-2007, op. cit., p. 93. 
L'attitude de Jean-Paul II avec les jeunes et la façon avec laquelle il communiquait avec eux étaient remarquables et souvent soulignées par des observateurs. J.-M. Lustiger, lui aussi, en admirant cette faculté du Saint Père, trouvait que pendant ces rencontres il était parfaitemnet libre et vrai et que cela ne trompait pas les jeunes ${ }^{14}$. Il était tout entier à sa mission. Il ne jouait pas au pape, mais il était le pape. Tout le monde l'a vu et l'a senti ${ }^{15}$. Le Saint Père pendant les rencontres avec les jeunes ne tombait jamais dans la démagogie, n'avait pas de recette apostolique, mais il agissait tout naturellement en pasteur, il aimait les jeunes. Cet amour était un acte typiquement pastoral, car il savait que le devoir de transmettre la Bonne Nouvelle aux générations suivantes était un impératif de la mission apostolique. En résumant théologiquement cette attitude, J.-M. Lustiger savait que ce souci de la jeunesse pour Jean-Paul II était lié à la condidtion présente de l'Eglise qui est en pèlerinage jusqu'à la venue de son Seigneur en gloire ${ }^{16}$.

Selon Jean-Marie Lustiger les voyages de Jean-Paul II étaient des événements qui portaient un caractère de spectacle. Mais tout le monde n'a pas vu le même spectacle, bien que tout le monde ait porté les yeux sur le même spectaculaire. Ces événements ressemblaient aux rencontres avec le Christ, car tous pouvaient le voir, mais seulement ceux qui voulaient croire, c'est-à-dire se laissaient pénétrer par le fond des choses, comprennaient le vrai sens de l'événement ${ }^{17}$. Ces voyages apostoliques ne découlaient pas uniquement d'une opportune utilisation des moyens de communication, mais ils étaient enracinés dans la nature missionaire de l'Eglise. Cette méthode ne manifestait pas seulement le charisme personnel de Jean-Paul II, mais une réalité ecclésiale fondamentale: la conjonction du ministère pastoral de successeur de Pierre avec la vocation missionnaire de Paul, l'apôtre des nations ${ }^{18}$.

${ }^{14}$ Voir: J.-M. Lustiger, 16 octobre: „Je ne sais pourquoi, j’ai éprouvé une immense joie”, en: Jean-Marie Lustiger, témoin de Jean-Paul II. Articles, conférences, entretiens, homélies... 19802007, op. cit., p. 14.

${ }^{15}$ Voir: J.-M. Lustiger, Le pape en France, en: Jean-Marie Lustiger, témoin de Jean-Paul II. Articles, conférences, entretiens, homélies... 1980-2007, op. cit., p. 24.

${ }^{16}$ Voir: J.-M. Lustiger, Successore di Petro e imitatore di Paulo, en: Giovanni Paulo II pellegrino per il Vangelo, ouvrage collectif, op. cit., p. 117.

${ }^{17}$ Voir: J.-M. Lustiger, L'Eglise en France révélée à elle-même, en: Le pape en France, JeanMarie Lustiger, témoin de Jean-Paul II. Articles, conférences, entretiens, homélies... 1980-2007, op. cit., p. 29.

${ }^{18}$ Voir: J.-M. Lustiger, Le successeur de Pierre, porteur du charisme de Paul, en: Le pape en France, Jean-Marie Lustiger, témoin de Jean-Paul II. Articles, conférences, entretiens, homélies... 1980-2007, op. cit., p. 47. 


\section{Méthodes}

La méthode pastorale de Jean-Paul II, la plus connue et la plus souvent accentuée, était le fait que pour lui chaque personne était très précieuse. Il savait manifester une singulière attention à chaque personne et la foule était pour lui une assemblée réunie sous ses yeux ${ }^{19}$. L'attention particulière qu'il portait à une personne n'exprimait pas uniquement une sympathie humaine, ni une richesse d'affectivité, ni des bonnes manières, mais elle était d'un autre ordre. Elle était un acte de foi et de reconnaissance de pasteur pour une de ses brebis entre mille ${ }^{20}$.

La stratégie de Jean Paul II interdisait de considérer l'homme comme un matériau soumis à d'aveugles déterminismes ${ }^{21}$. Il a élaboré les éléments constitutifs de la condition humaine non à la base des schémas interprétatifs de dialectique du pouvoir, de luttes des classes, mais en vivant le drame d'une humanité oppressée et méprisée. Les appels : «N'ayez pas peur» et «Ouvrez la porte aux Christ» montraient comment lui-méme avait exorcisé cette peur par la force de la raison et de la foi.

Il faut souligner l'optimisme de Jean-Paul II, si présent dans le message de l'espérance de Vatican II, qui s'appuyait sur la mise en oeuvre du message de l'Evangile. Beaucoup de politiques ont été déconcertés par cet optimisme car ils n'ont pas compris le coeur de son attitude et faussement ils parlaient de la «politique de Jean-Paul II»" ${ }^{22}$. Jean-Paul II, en dialoguant avec les personnalités les plus diverses de la politique, de l'économie et de la culture, dont beaucoup étaient des agnostiques, des incroyants ou des athées, n'engageait pas uniquement la cordialité qui certainement ajoutait à la profondeur de ses rencontres tant appréciées par ses interlocuteurs, mais toutes ses rencontres étaient vécues dans la vision éclairée par la foi de l'humanité23.

Les homélies de Jean-Paul II portaient une énergie toujours neuve grâce à son langage, qui n'exprimait pas des propos généreux et rebattus, mais c'était

${ }^{19}$ Voir: J.-M. Successore di Petro e imitatore di Paulo, en: Giovanni Paulo II pellegrino per il Vangelo, ouvrage collectif, op. cit., p. 116-117.

${ }^{20}$ Voir: J.-M. Lustiger, Le successeur de Pierre, porteur du charisme de Paul, en: Le pape en France, Jean-Marie Lustiger, témoin de Jean-Paul II. Articles, conférences, entretiens, homélies. . 1980-2007, op. cit., p. 44.

${ }^{21}$ Voir: J.-M. Lustiger, La puissance visionnaire du serviteur de la Vérité, „Géopolitique“ (1997) n $n^{\circ} 58$, p. 3.

${ }^{22}$ Voir: J.-M. Lustiger, La force d'une pensée éclairée par la foi, en: Jean-Marie Lustiger, témoin de Jean-Paul II. Articles, conférences, entretiens, homélies... 1980-2007, op. cit., p. 69.

${ }^{23}$ Voir: J.-M. Lustiger, Le pape du concile, en: Jean-Marie Lustiger, témoin de Jean-Paul II. Articles, conférences, entretiens, homélies... 1980-2007, op. cit., p. 108. 
un longage nouveau sur l'homme et sa vie qui découlait de la foi et de la Parole de Dieu sur l'homme ${ }^{24}$. Jean-Paul II a apporté une nouvelle façon de parler de l'homme à laquelle la scène politique et culturelle occidentale n'était pas habituée. C'était un langage tenu à la lumière du Christ basé sur une pensée personnaliste et doté d'une méthode phénoménologique. Pour Jean-Paul II la base de la culture se trouvait dans l'homme et dans sa liberté spirituelle ${ }^{25}$. Dans son enseignement on trouve une nouveauté linguistique qui consiste dans le fait que le Saint Père ne parlait pas de la nature humaine d'une manière abstraite, ni de l'humanité comme d'un collectif, mais de l'homme concret et historique ${ }^{26}$. L'effort personnel de Jean-Paul II pour célébrer dans la langue du pays qu'il visitait était hautement significatif car l'unité catholique ne se réalise pas par l'imposition d'une seule langue qui prétendrait être universelle ${ }^{27}$.

Jean-Paul II posait des questions fondamentales. Même si les auditeurs le guettaient sur ses réponses, le Saint Père ne répondait pas. Il posait au nom du Christ les questions capitales que jamais personne ne leur posait, pour les obligait à se mettre en chemin sûrement austère mais nécessaire et purificateur ${ }^{28}$. En parlant de la civilisation de la mort, il ne voulait pas déconsidérer tout progès humain, mais il voulait exposer la civilisation de l'amour que les hommes doivent construire. C'était sa façon de dire, que le christianisme vit toujours sa jeunesse et de souligner d'une manière saisissante son actualitée ${ }^{2}$.

Notre civilisation se caractérise par une rupture entre les générations. JeanMarie Lustiger était convaincu, que cette cassure concerne aussi la transmission de la foi. Jean-Paul II qui, en s'appuant sur la Parole de Dieu, a trouvé une solution à ce problème. Il parlait de la logique spirituelle interne de l'Eglise, qui consiste dans le fait, que chaque événement salvifique est inscrit dans le présent de chaque génération ${ }^{30}$.

${ }^{24}$ Voir: J.-M. Lustiger, Le pape en France, en: Jean-Marie Lustiger, témoin de Jean-Paul II. Articles, conférences, entretiens, homélies... 1980-2007, op. cit., p. 24.

${ }^{25}$ Voir: J.-M. Lustiger, L'homme au ceour de la culture, en: Jean-Marie Lustiger, témoin de Jean-Paul II. Articles, conférences, entretiens, homélies... 1980-2007, op. cit., p. 72-73.

${ }^{26}$ Voir: J.-M. Lustiger, L’homme au ceour de la culture, en: Jean-Marie Lustiger, témoin de Jean-Paul II. Articles, conférences, entretiens, homélies... 1980-2007, op. cit., p. 76.

${ }^{27}$ Voir: J.-M. Lustiger, Le pape du concile, en: Jean-Marie Lustiger, témoin de Jean-Paul II. Articles, conférences, entretiens, homélies... 1980-2007, op. cit., p. 117.

${ }^{28}$ Voir: J.-M. Lustiger, Le pape en France, en: Jean-Marie Lustiger, témoin de Jean-Paul II. Articles, conférences, entretiens, homélies... 1980-2007, op. cit., p. 26.

${ }^{29}$ Voir: J.-M. Lustiger, Le „nouvel âge de l'humanité“, en: Jean-Marie Lustiger, témoin de Jean-Paul II. Articles, conférences, entretiens, homélies... 1980-2007, op. cit., p. 91.

${ }^{30}$ Voir: J.-M. Lustiger, Jean-Paul II et l'Eglise. Une pédagogie spirituelle, „Christus“ (2005) $n^{0} 208$, p. 494. 
Dès le début de son pontificat, Jean-Paul II consacrait une grande part de son temps à visiter les Eglises locales du monde entier. Il a surpris les habitants de Rome qui craignaient que le nouveau pape polonais ne leur impose le mode polonais de vie chrétienne, en leur demandant de l'accueillir au nom du Seigneur comme ils ont accueilli ses prédécesseurs ${ }^{31}$. En exerçant sa mission de pasteur suprême de l'Eglise, il allait à la rencontre de ses frères dans l'éspiscopat pour les confirmer dans la foi, comme Jésus l'a commandé à Pierre ${ }^{32}$. Il était avec eux pour les aider à remplir leur propre mission de pasteur ${ }^{33}$. Jean-Paul II n'allait pas en visite pastorale dans une Eglise locale, que s'il y était invité par le pasteur de cette Eglise et il préparait soigneusement chaque visite avec les évêques de la région. En mettant en oeuvre la collégialité, il ne se présentait jamais comme le pape face à une Eglise particulière, mais comme un successeur de Pierre qui, en union avec les évêques du lieu, annonçait à une portion du troupeau le mystère du Christ $t^{34}$. Il portait les Eglises locales dans sa prière et il était pour eux un témoin privilégié du don de grâce offert à chacune des nations où l'Evangile du Christ avait été annoncé et où l'Eglise du Christ existait ${ }^{35}$. Chacune des visites pastorales avait comme but de déployer les richesse de la foi et d'aider les Eglises à exprimer leurs faiblesses pour vivre le pardon et la réconciliation. Jean-Paul II venait pour consoler les Eglises, pour montrer l'homme et pour faire une catéchèse itinérante ${ }^{36}$. Chacun des voyages apostoliques de Jean-Paul II traçait dans une Eglise un chemin spirituel dont les fruits étaient imprévisibles ${ }^{37}$. Il savait traduire en action les réalités juridiques de la primauté et de la collegialitée ${ }^{38}$.

${ }^{31}$ Voir: J.-M. Lustiger, Le successeur de Pierre, porteur du charisme de Paul, en: Le pape en France, Jean-Marie Lustiger, témoin de Jean-Paul II. Articles, conférences, entretiens, homélies... 1980-2007, op. cit., p. 37-38.

${ }^{32}$ Voir: Luc 22,32.

${ }^{33}$ Voir: J.-M. Lustiger, Le successeur de Pierre, porteur du charisme de Paul, en: Le pape en France, Jean-Marie Lustiger, témoin de Jean-Paul II. Articles, conférences, entretiens, homélies... 1980-2007, op. cit., p. 45.

${ }^{34}$ Voir: J.-M. Lustiger, Successore di Petro e imitatore di Paulo, en: Giovanni Paulo II pellegrino per il Vangelo, ouvrage collectif, op. cit., p. 119-120.

${ }^{35}$ Voir: J.-M. Lustiger, Le successeur de Pierre, porteur du charisme de Paul, en: Le pape en France, Jean-Marie Lustiger, témoin de Jean-Paul II. Articles, conférences, entretiens, homélies... 1980-2007, op. cit., p. 48.

${ }^{36}$ Voir: J.-M. Lustiger, Successore di Petro e imitatore di Paulo, en: Giovanni Paulo II pellegrino per il Vangelo, ouvrage collectif, op. cit., p. 122-123.

${ }^{37}$ Voir: J.-M. Lustiger, Le successeur de Pierre, porteur du charisme de Paul, en: Jean-Marie Lustiger, témoin de Jean-Paul II. Articles, conférences, entretiens, homélies... 1980-2007, op. cit., p. 54.

${ }^{38}$ Voir: J.-M. Lustiger, Le pape du concile, en: Jean-Marie Lustiger, témoin de Jean-Paul II. Articles, conférences, entretiens, homélies... 1980-2007, op. cit., p. 119. 
Il faut souligner les canonisations de témoins de l'amour de Dieu comme une démonstration éclatante qui se déroulait dans les pays où ces saints ont vécu et témoigné de la foi. Même si la visite apostolique n'était pas accompagnée d'une canonisation, sa démarche était toujours un acte de foi en ce que Dieu accomplissait dans chaque Eglise particulière ${ }^{39}$. Des nombreuses canonisations exprimaient l'ecclésiologie de Jean-Paul II qui s'appuyait particulièrement sur la vocation à la sainteté comme vocation baptismale de tous les chrétiens ${ }^{40}$.

Certaines personnes reprochaient au Saint Père d'agir selon les mécanismes communistes, car il a été élevé dans ce système. J.-M. Lustiger considérait cela comme absurde. Sachant ce que sont les appareils dans le système du communisme, et connaissant le communisme réel, Jean-Paul II était absolument le contraire d'un homme d'appareil. Dans le gouvernement de l'Eglise il agissait en homme libre, en prenant les choses avec la hauteur de vue de la foi et en même temps avec le réalisme de la charitée ${ }^{41}$. Loin d'être crispé sur les schémas traditionnels qui «de gauche» comme «de droite» récusaient a priori toute innovation, il était un pasteur d'ouverture et de nouveauté évangélique ${ }^{42}$.

Selon Jean-Marie Lustiger, Jean-Paul II avait une véritable pédagogie spirituelle, laquelle était une solution pour la crise dans l'Eglise. Ses trois premières encycliques sur le Christ Rédempteur, sur Dieu miséricordieux et sur l'Esprit Saint, et ensuite les trois exhortations post-synodales au sujet des prêtres, des laïcs et des religieux, rappelaient qu'il faut se mettre tout d'abord sous le regard du Christ, de Dieu miséricrdieux et de l'Esprit Saint pour pouvoir discerner ce que le Seigneur demande à l'Eglise dans une situation de crise ${ }^{43}$. Il faut mettre aussi dans la méme perspective l'enseignement du Saint Père au sujet de la vocation sacerdotale et religieuse tellement important pour JeanMarie Lustiger. Jean-Paul II, dans le contexte de la crise des vocations et de la discussion au sujet du célibat dans la lignée de Vatican II, a demandé aux prêtres et à toutes les personnes consacrées de se mettre à nouveau au coeur du mystère

${ }^{39}$ Voir: J.-M. Lustiger, Le successeur de Pierre, porteur du charisme de Paul, en: Le pape en France, Jean-Marie Lustiger, témoin de Jean-Paul II. Articles, conférences, entretiens, homélies... 1980-2007, op. cit., p. 49.

${ }^{40}$ Voir: J.-M. Lustiger, Jean-Paul II et l'Eglise. Une pédagogie spirituelle, „Christus“ (2005) no 208, p. 497.

${ }^{41}$ Voir: J.-M. Lustiger, La force d'une pensée éclairée par la foi, en: Jean-Marie Lustiger, témoin de Jean-Paul II. Articles, conférences, entretiens, homélies... 1980-2007, op. cit., p. 67.

${ }^{42}$ Voir: J.-M. Lustiger, La puissance visionnaire du serviteur de la Vérité, „Géopolitique“ (1997) n n 58, p. 5.

${ }^{43}$ Voir: J.-M. Lustiger, Jean-Paul II et l'Eglise. Une pédagogie spirituelle, „Christus“ (2005) $n^{\circ} 208$, p. 495-496. 
de leur existence et de leur vocation et il les a invités à formuler leurs oppinions comme le Christ le demande dans l'Evangile ${ }^{44}$, non selon le monde mais selon l'Esprit Saint ${ }^{45}$.

\section{Efficacité}

D'où venait ce «succès» apostolique de Jean-Paul II? J.-M. Lustiger, en préparant ses diocésains à la venue de Jean-Paul II en France, les a conduits à Jérusalem, au jour de la Pentecôte, pour écouter parler Pierre. C'est parce que l'Esprit de Dieu parlait par la bouche des Apôtres, que ce qui paraissait impossible se réalisait et émerveillait les auditeurs ${ }^{46}$. Lorsque le Saint Père acceptait une invitation faite par une Eglise locale, on pressentait une conduite de l'Esprit Saint à l'oeuvre dans cette Eglise ${ }^{47}$.

Jean-Paul II était une personne médiatique. Sa personnalité intéressait, séduisait et fascinait, car tout en lui - sa personne, ses dons, son histoire - était mis au service de la mission, que Dieu lui a confiée ${ }^{48}$. L'attitude pastorale de Jean-Paul II ne s'exprimait que par un acte de foi dans la fonction de pasteur. Elle trouvait son fondement dans la fidélité à la parole do Jésus : «Pais mes brebis» ${ }^{49}$.

Il respectait chaque parsonne. Aux questions posées par des jeunes, étonnantes de vérité et exigeantes pour la foi, Jean-Paul II répondait toujours scrupuleusement. Le pape ne faisait jamais ni concession ni démagogie, mais paisiblement, avec une allégresse visible et communicative, répondait en Jésus-Christ ${ }^{50}$.

L'efficacité de la prédication de Jean-Paul II découlait du fait, qu'il parlait au nom de l'homme qui ne peut se comprendre seulement à partir de lui-seul, et que le Christ assume et achève. Son message était fondé sur l'expérience de sa vie et

${ }^{44}$ Voir: Jn 7,24; 8,15-16.

${ }^{45}$ Voir: J.-M. Lustiger, Les prêtres, la vie consacrée et les vocations, en: Jean-Marie Lustiger, témoin de Jean-Paul II. Articles, conférences, entretiens, homélies... 1980-2007, op. cit., p. 124.

${ }^{46}$ Voir: J.-M. Lustiger, Message pour la Pentecôte, en: Jean-Marie Lustiger, témoin de JeanPaul II. Articles, conférences, entretiens, homélies... 1980-2007, op. cit., p. 18.

${ }^{47}$ Voir: J.-M. Lustiger, Le successeur de Pierre, porteur du charisme de Paul, en: Jean-Marie Lustiger, témoin de Jean-Paul II. Articles, conférences, entretiens, homélies... 1980-2007, op. cit., p. 52 .

${ }^{48}$ Voir: J.-M. Lustiger, Tout entier au service de sa mission, en: Le pape en France, Jean-Marie Lustiger, témoin de Jean-Paul II. Articles, conférences, entretiens, homélies... 1980-2007, op. cit., p. 35 .

${ }^{49} \mathrm{~J} 21,15$.

${ }^{50}$ Voir: J.-M. Lustiger, Le pape en France, en: Jean-Marie Lustiger, témoin de Jean-Paul II. Articles, conférences, entretiens, homélies... 1980-2007, op. cit., p. 26. 
il attestait que l'Eglise ne voit pas d'abord l'ennemi, mais elle voit un être perdu, menacé de la ruine de son identités1.

Il fut le premier pape vraimenet moderne, parce qu'il connaissait la modernité en tant que philosophe phénoménologue et en tant que pasteur qui a dû et a su lutter pour affirmer son identité et sa liberté spirituelle ${ }^{52}$. Il voulait communiquer à tous et à chacun le secret de sa joie et de sa sérénité, c'est-à-dire sa foi dans le Christ et son amour pour Lui. De sa foi personnelle découlait l'enthousiasme de son ministère évangélisateur, car il désirait que la foi pénètre toutes les dimensions sociales de l'existence humaine, y compris la culture et les loisirs ${ }^{53}$.

Son témoignage dans les dernières années de sa vie était, à cause de sa faiblesse physique, plus fort qu'avant ${ }^{54}$. Jean-Marie Lustiger était convaincu que Jean-Paul II dans sa souffrance accomplissait sa mission de successeur de Pierre en plénitude. Même si aux yeux du monde il ne remplissait plus ses fonctions comme auparavant, c'était à ce moment-là, selon la parole de Jésus ${ }^{55}$, qu'il allait glorifier Dieu comme l'apôtre Pierre ${ }^{56}$.

Jean-Marie Lustiger a constaté le renversement radical de persperctive dans l'enseignement et dans l'action de Jean-Paul II. Selon lui le pape pour relever le défi des temps nouveaux, pour évaluer les besoins réels et apporter des réponses aux questions de l'humanité, n'a choisi comme champ de réflexion ni la politique, ni la sociologie, ni l'anthropologie, mais s'est adressé à l'homme blessé et racheté, que l'on peut aimer grâce à la foi ${ }^{57}$.

${ }^{51}$ Voir: J.-M. Lustiger, Le pape du concile, en: Jean-Marie Lustiger, témoin de Jean-Paul II. Articles, conférences, entretiens, homélies... 1980-2007, op. cit., p. 107.

${ }^{52}$ Voir: J.-M. Lustiger, L’Eglise en France révélée à elle-même, en: Le pape en France, JeanMarie Lustiger, témoin de Jean-Paul II. Articles, conférences, entretiens, homélies... 1980-2007, op. cit., p. 30-31.

${ }^{53}$ Voir: J.-M. Lustiger, Le successeur de Pierre, porteur du charisme de Paul, en: Le pape en France, Jean-Marie Lustiger, témoin de Jean-Paul II. Articles, conférences, entretiens, homélies... 1980-2007, op. cit., p. 39.

${ }^{54}$ Voir: J.-M. Lustiger, Le courage de la vérité, en: Jean-Marie Lustiger, témoin de Jean-Paul II. Articles, conférences, entretiens, homélies... 1980-2007, op. cit., p. 170.

${ }^{55}$ Voir: J 21,19.

${ }^{56}$ Voir: J.-M. Lustiger, A la suite de Bartimée et de Mère Teresa, en: Jean-Marie Lustiger, témoin de Jean-Paul II. Articles, conférences, entretiens, homélies... 1980-2007, op. cit., p. 173-174.

${ }^{57}$ Voir: J.-M. Lustiger, Les prêtres, la vie consacrée et les vocations sous le Pontificat de JeanPaul II, „L'Osservatore Romano“ éditions française, nº 45, p. 11. 


\section{Bibliography}

Jean-Marie Lustiger, témoin de Jean-Paul II. Articles, conférences, entretiens, homélies... 1980-2007, Paris 2011, édition Parole et Silence, Communio.

Jean-Paul II et l'Eglise. Une pédagogie spirituelle, „Christus“ (2005) n 208, p. 492-498.

La puissance visionnaire du serviteur de la Vérité, „Géopolitique“ (1997) n 58, p. 2-7.

Successore di Petro e imitatore di Paulo, en: Giovanni Paulo II pellegrino per il Vangelo, ouvrage collectif, Milan 1988, edizioni Paoline - editrice SAIE, p. 115-124.

Les prêtres, la vie consacrée et les vocations sous le Pontificat de Jean-Paul II, „L'Osservatore romano“ éditions française, $\mathrm{n}^{\circ} 45$, p. 9 et 11; $\mathrm{n}^{\circ}$ 46, p. 9-10. 